\title{
How Toxic is Milkweed when Harvested and Cooked according to Myaamia Tradition?
}

\author{
Michael A. Everest ${ }^{1}$, Michael P. Gonella ${ }^{2}$, Holly G. Bowler ${ }^{1}$, and Joshua R. Waschak ${ }^{1}$ \\ ${ }^{1}$ Department of Chemistry, Westmont College, Santa Barbara, USA. ${ }^{2}$ Department of Environmental Horticulture, Santa \\ Barbara City College, Santa Barbara, USA. \\ *meverest@westmont.edu
}

\begin{abstract}
Asclepias syriaca L. (common milkweed) is known to contain sufficient amounts of cardiac glycosides, which are known to be toxic to humans. Nonetheless, it is traditionally used for food by Native Americans, including the Myaamia people of Indiana and Oklahoma. In order to test the hypothesis that traditional horticultural and culinary practices prevent the Myaamia from ingesting toxic levels of cardiac glycosides, we have determined the level of glycosides (digitoxin equivalent) in $A$. syriaca 1 ) in various parts of the plant, 2) at various heights for pre-reproductive plants, and 3) before and after cooking according to traditional Myaamia procedures. Plants were grown, harvested, dried, ground, and extracted twice with ethanol. The amount of digitoxin-equivalent glycoside in plant extract was determined spectrophotometrically using 2,2',4,4'-tetranitrodiphenyl, a selective derivatizing agent. We find that all parts of the plant contain significant levels of cardiac glycosides at all stages of growth. Plants harvested as young shoots for food, the common practice of the Myaamia, contain slightly lower levels of cardiac glycosides when compared to the leaves and stems of older, taller plants. Moreover, the toxicity is significantly reduced by the traditional Myaamia cooking procedure-a repeated boiling with several changes of water. Therefore, it appears as though the risk of glycoside poisoning from traditional Myaamia use of milkweed for food is moderated by their harvesting practice and traditional cooking procedure.
\end{abstract}

Received January 4, 2019

OPENӘACCESS

Accepted May 7, 2019

10.1.2019.1487

Published August 6, 2019

Keywords Botany, Cardiac glycosides, Toxins, Food plants, Food preparation

Copyright (C) 2019 by the author(s); licensee Society of Ethnobiology. This is an open-access article distributed under the terms of the Creative Commons Attribution-NonCommercial 4.0 International Public License (https://creativecommons.org/licenses/by-nc/4.0), which permits non-commercial use, distribution, and reproduction in any medium, provided the original author and source are credited.

\section{Background}

Asclepias syriaca (common milkweed) grows ubiquitously in the midwestern United States and has many established and potential uses (Gaertner 1979). For example, one $A$. syriaca latex-related compound was found to have cytotoxic effects with breast cancer cells (Araya et al. 2012). Schlegel et al. (2011) also suggested numerous reported beneficial nutritional uses of $A$. syriaca.

In fact, $A$. syriaca has been used for centuries by native North Americans for fiber, medicine, and food (Cheatham and Johnston 1995; Moerman 1988; Whitford 1941). Native groups using milkweed for food include, but are not limited to the Tsalagi, Anishinaabe, Haudenoshonee, Lakota, Menominee, and Myaamia (Densmore 1974; Gilmore 1911; Gonella 2007; Hamel and Chilotsky 1975). We are particularly interested in the cultural practices of the Myaamia. They are historically centered in northern Indiana, and many still live there. Many Myaamia people also live in northeastern Oklahoma on reservation lands. The Miami Tribe of Oklahoma is the seat of the federally-recognized tribal government.

Despite the fact that $A$. syriaca is believed to contain toxic levels of cardiac glycosides, contemporary Myaamia people continue to harvest wild, young shoots of milkweed, prepare them by traditional methods passed down through generations, and use them as food without any known ill effects (Gonella 2007). Myaamia elders mention no negative effects, but instead describe health benefits of eating milkweed after a long, sedentary winter, and report enjoying the taste of milkweed cooked much like asparagus. 
The Myaamia have highly specific cultural practices pertaining to the use $A$. syriaca for food. Specifically, they only harvest early spring shoots less than $25 \mathrm{~cm}$ in height and with more than four leaves. To cook, they boil the shoots, changing the water more than once (Gonella 2007). Due to the specificity of harvesting methods used by the Myaamia, including exact time of harvest and stage of growth harvested, and the lack of any known negative health effects, we hypothesized that traditional harvesting and preparation methods allow them to avoid toxic levels of cardiac glycosides, thereby rendering this otherwise toxic plant nutritious. To test this hypothesis, we determined the amount of digitoxinequivalent cardiac glycosides in $A$. syriaca 1$)$ in various parts of the plant, 2) at various heights, and 3) before and after cooking according to traditional Myaamia procedures.

\section{Methods}

Plant Growth and Collection

Seeds were collected along public roadsides in northeastern Indiana. A. syriaca is not present on Myaamia reservation lands in Oklahoma due to its intentional eradication by non-Myaamia farmers who owned the land before Myaamia Tribe ownership, and who considered it a noxious weed. An herbarium specimen for $A$. syriaca is housed at the Miami Tribe of Oklahoma archives in Miami, Oklahoma.

For the studies comparing different parts of raw plant material, seeds were planted in Sunshine Growth Media \#4, germinated in a greenhouse on the Santa Barbara City College campus, and maintained at $24.2^{\circ} \mathrm{C}$ until leaves appeared. Seedlings were then placed in outdoor, raised, experimental plots of similar solar exposure, soil type, and watering regime. Plants were collected whole, and excess dirt (and insects, where applicable) was removed manually. The mid-height of each plant was ascertained, to analyze the "upper" and "lower" portions separately.

\section{Cooking A. syriaca}

Glycoside levels of cooked $A$. syriaca shoots were measured using 20 immature (pre-reproductive), newly emerging shoots of wild plants collected along public roadsides in northeastern Indiana. These shoots were selected according to Myaamia practice: only shoots less than $25 \mathrm{~cm}$ high and having no more than 4 leaves were harvested. The shoots were weighed, boiled twice for ten minutes, with fresh water added for the second boil (Gonella 2007).
Excess water was shaken off, and cooked plant material was weighed wet.

\section{Plant Preparation}

For raw and cooked samples, plant specimens were dried in an oven at $45-60^{\circ} \mathrm{C}$ for approximately 24 hours, ground to a fineness of 60 grit in a mill grinder, and stored in sealed glass vials. We initially used an extraction procedure similar to that of Rasmann et al. (2009), but significant variation in results caused us to suspect that the extraction procedure was not removing all the glycosides from the plant material. After testing several different methods, we performed extraction as follows: 40-100 $\mathrm{mg}$ of ground plant material was suspended in $10.00 \mathrm{ml}$ of ethanol in a 50 $\mathrm{mL}$ Erlenmeyer flask and shaken overnight in an incubator shaker maintained at $50^{\circ} \mathrm{C}$. The solid plant material was separated from the extract by vacuum filtration, and resuspended in $10.00 \mathrm{ml}$ of fresh ethanol. This second suspension was shaken again overnight at $50^{\circ} \mathrm{C}$, filtered, and the two extractions were combined and diluted to $25.00 \mathrm{ml}$ with ethanol. In some test trials, a third extraction showed measurable, but minimal, amounts of glycoside remain in the plant material after two extractions. Moreover, this double-extraction procedure yielded similar results, but required much less material, when compared to plants extensively extracted with ethanol in a vacuum Soxhlet apparatus. Ethanol was either 90\% anhydrous, denatured, spectrophotometric grade; 200 proof ACS reagent grade; or 190 proof HPLC grade.

\section{Determination of Glycosides}

The amount of cardiac glycosides present in the plant extract was determined with a spectrophotometric assay in which the glycosides were selectively derivatized with 2,2',4,4'-tetranitrodiphenyl (TNDP), resulting in an intensely colored Meisenheimer complex (Brower et al. 1972; Rasmann et al. 2009). TNDP was synthesized according to the procedure reported by Brower et al. (1972). For the determination itself, $675 \mu \mathrm{L}$ of plant extract, $1.00 \mathrm{~mL}$ of $0.1 \mathrm{M}$ $\mathrm{NaOH}$, and $1.00 \mathrm{~mL}$ of ethanol (blank) or $1.00 \mathrm{~mL}$ of plant extract (sample) were added to a small test tube, rapidly mixed, and incubated in a $25^{\circ} \mathrm{C}$ water bath for ten minutes. The absorbance of the blank and sample solutions was determined in a Nicolet Evolution 300 $\mathrm{UV} / \mathrm{Vis}$ spectrophotometer at $618 \mathrm{~nm}$, the peak of the Meisenheimer complex absorption. Standards were prepared from pure digitoxin in ethanol. They 


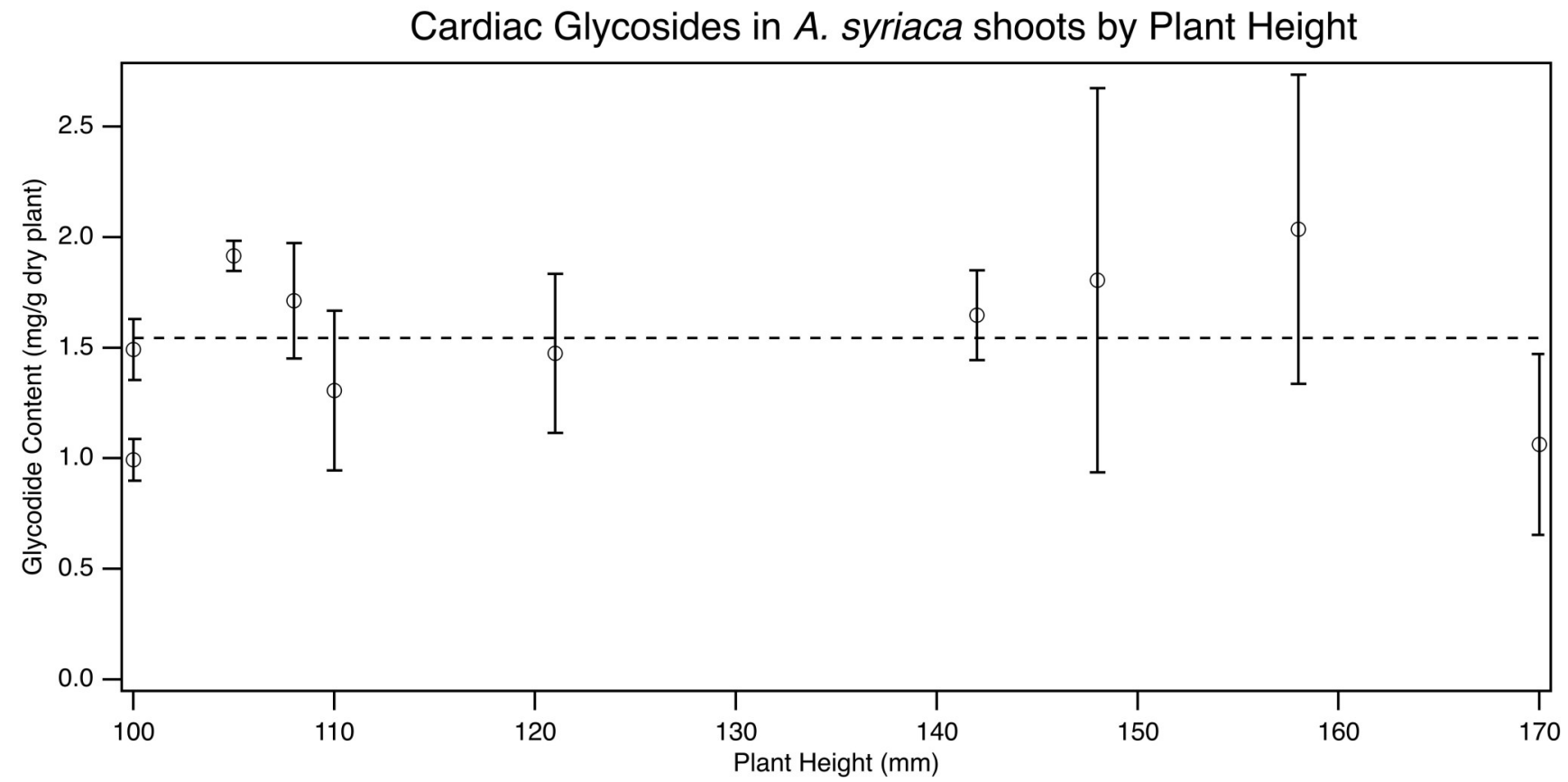

Figure 1 Digitoxin-equivalent cardiac glycoside content in shoots of A. syriaca as it depends on shoot height. Error bars are the standard deviation for five measurements on each plant. The horizontal line at $1.5 \mathrm{mg} / \mathrm{g}$ indicates the average cardiac glycoside abundance in these shoots.

were treated in the same way as the plant extracts in the spectrophotometric assay. Therefore, although the botanical samples contain several different cardiac glycosides, we assume that TNDP forms a complex with all of them, that these complexes all have the same extinction coefficient as the digitoxin complex, and we therefore report digitoxin-equivalent cardiac glycoside.

We initially attempted to perform the analysis on a small scale in 96 well plates, as has been demonstrated by Rasmann et al. (2009), but we found that the well plates were rapidly and inconsistently fouled by a white substance (likely related to plant latex), which made the results irreproducible. Even in the procedure described above, white deposits were frequently observed on the cuvette walls, and the cuvettes had to be frequently and meticulously cleaned. Such deposits were never observed on the digitoxin standard samples.

\section{Results}

Glycoside Abundance vs. Immature Plant Height

Ten immature (pre-reproductive) plants were harvested at several stages of growth having heights between 100 and $170 \mathrm{~mm}$. As shown in Figure 1, the amount of digitoxin-equivalent glycoside per dry plant material was $1.5 \pm 0.5 \mathrm{mg} / \mathrm{g}$ (reported error is the standard deviation of five measurements each on 10 plants). The correlation coefficient from linear regression was 0.02 , strongly suggesting that amount of glycoside is uncorrelated with plant height. This is a very small sample size for biological specimens that may include significant natural variability, so we cannot completely rule out some correlation in a large population of plants, but plant height does not appear to be a major factor in cardiac glycoside abundance for young plants.

\section{Glycoside Abundance vs. Part of Plant}

Several mature plants were separated into various parts: rhizomes, stems (upper and lower), leaves (upper and lower), and actively growing tops. The amount of cardiac glycoside in the plant material was determined separately for each section, and averaged across all plants. The results are displayed in Figure 2. Each plant was extracted once, and each extract was measured four times. For example, we had upper leaves from four plants, extracted each plant once, and performed the complexation reaction and spectrophotometric analysis four separate times on each extract for a total of sixteen measurements. The 


\section{Cardiac Glycosides in Various Parts of $A$. syriaca}

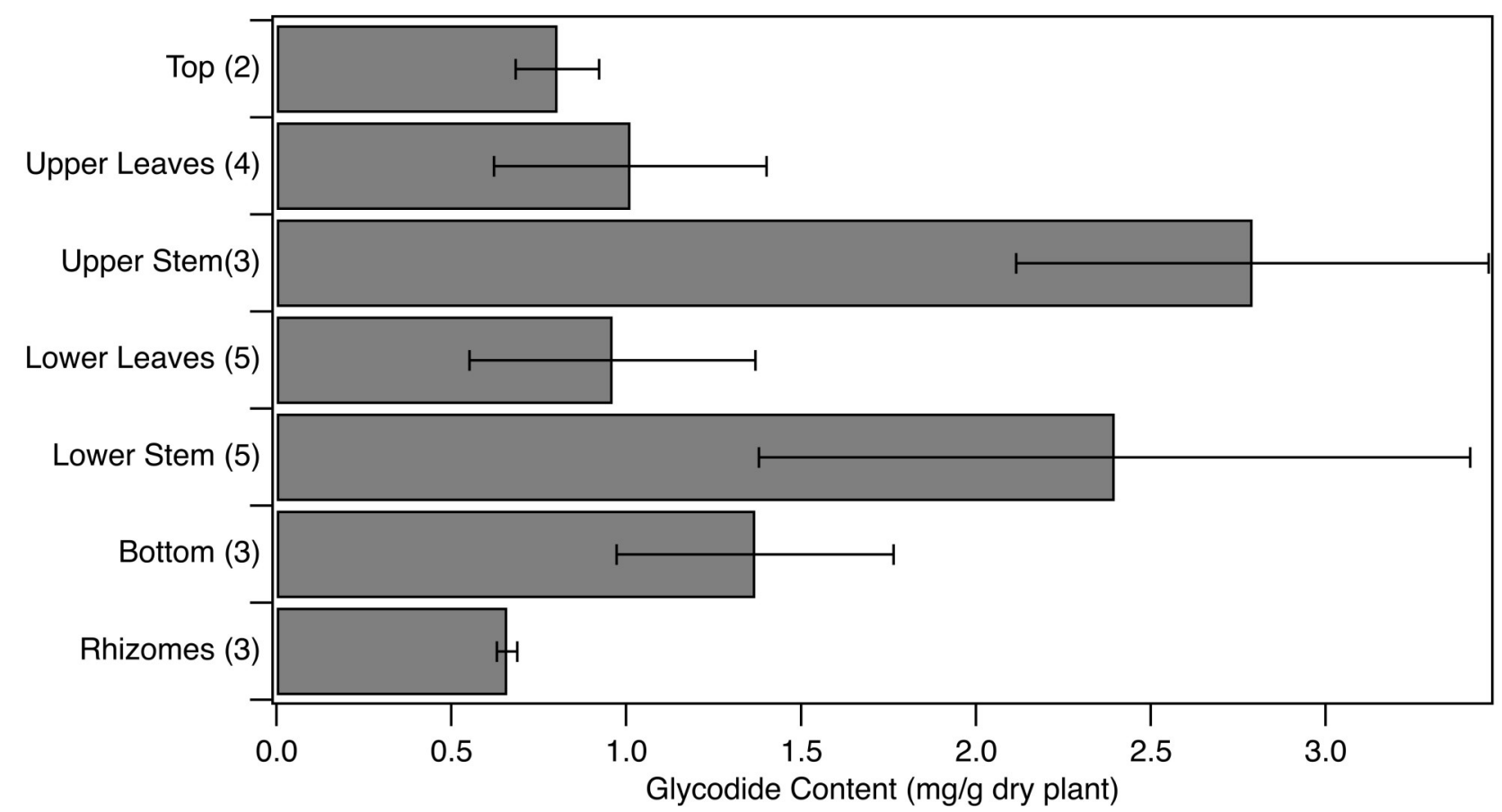

Figure 2 Amount of digitoxin-equivalent cardiac glycosides in various parts of $A$. syriaca. Values given are mg digitoxinequivalent cardiac glycoside per gram dried plant material. The number of different plants measured for each part is given in parenthesis. Error bars indicate standard deviation of the average measurements for each plant.

variance in the data between plants was comparable to the variance of the four measurements for each plant.

The stems tended to have the highest glycoside levels at approximately $2.5 \mathrm{mg} / \mathrm{g}$ dry plant, the leaves and tops had lower glycoside levels at approximately $1.0 \mathrm{mg} / \mathrm{g}$. We also measured the glycoside levels in the flowers from one plant, and found it to be $0.65 \pm 0.03 \mathrm{mg} / \mathrm{g}$ dry plant (error is standard deviation of four measurements). Rhizomes had the lowest glycoside levels at just over $0.5 \mathrm{mg} / \mathrm{g}$. All reported values are $\mathrm{mg}$ digitoxin-equivalent cardiac glycoside per gram dry plant material. The measured rhizome and stem glycoside levels are somewhat lower than values previously reported for "roots" and "shoots" by Rasmann et al. (2009), who found values closer to $4-5 \mathrm{mg} / \mathrm{g}$. It is currently unclear whether this discrepancy is owing to differences in the plants themselves or differences in the measurement protocol.

In light of Figure 2, it is interesting to note that the shoots measured, above (Figure 1), have glycoside levels intermediate between the actively growing tops and the stems of tall plants.

\section{Effect of Cooking on Glycoside Abundance}

Finally, the amount of cardiac glycoside was determined in plant material before and after cooking according to traditional Myaamia procedure. The amount of cardiac glycoside in cooked and uncooked immature plants is displayed in Figure 3. After drying, uncooked $A$. syriaca shoots contained $1.44 \pm 0.14 \mathrm{mg} /$ $\mathrm{g}$ dry plant material and cooked shoots contained $1.1 \pm 0.1 \mathrm{mg} / \mathrm{g}$ dry plant material. Therefore, roughly $25 \%$ of the original glycoside content is removed by repeatedly boiling in fresh water. Cooked shoots contain a significantly higher percentage of moisture $(90 \%)$ when compared to uncooked shoots $(85 \%)$. Therefore, owing to the greater water content in the cooked shoots, the amount of cardiac glycoside per gram wet plant decreases even more than the amount of cardiac glycoside per gram dried plant upon cooking: $0.20 \pm 0.03 \mathrm{mg} / \mathrm{g}$ for uncooked and $0.09 \pm$ $0.02 \mathrm{mg} / \mathrm{g}$ cooked. For these results, the reported error is the $95 \%$ C.I. for a total of 40 measurements 


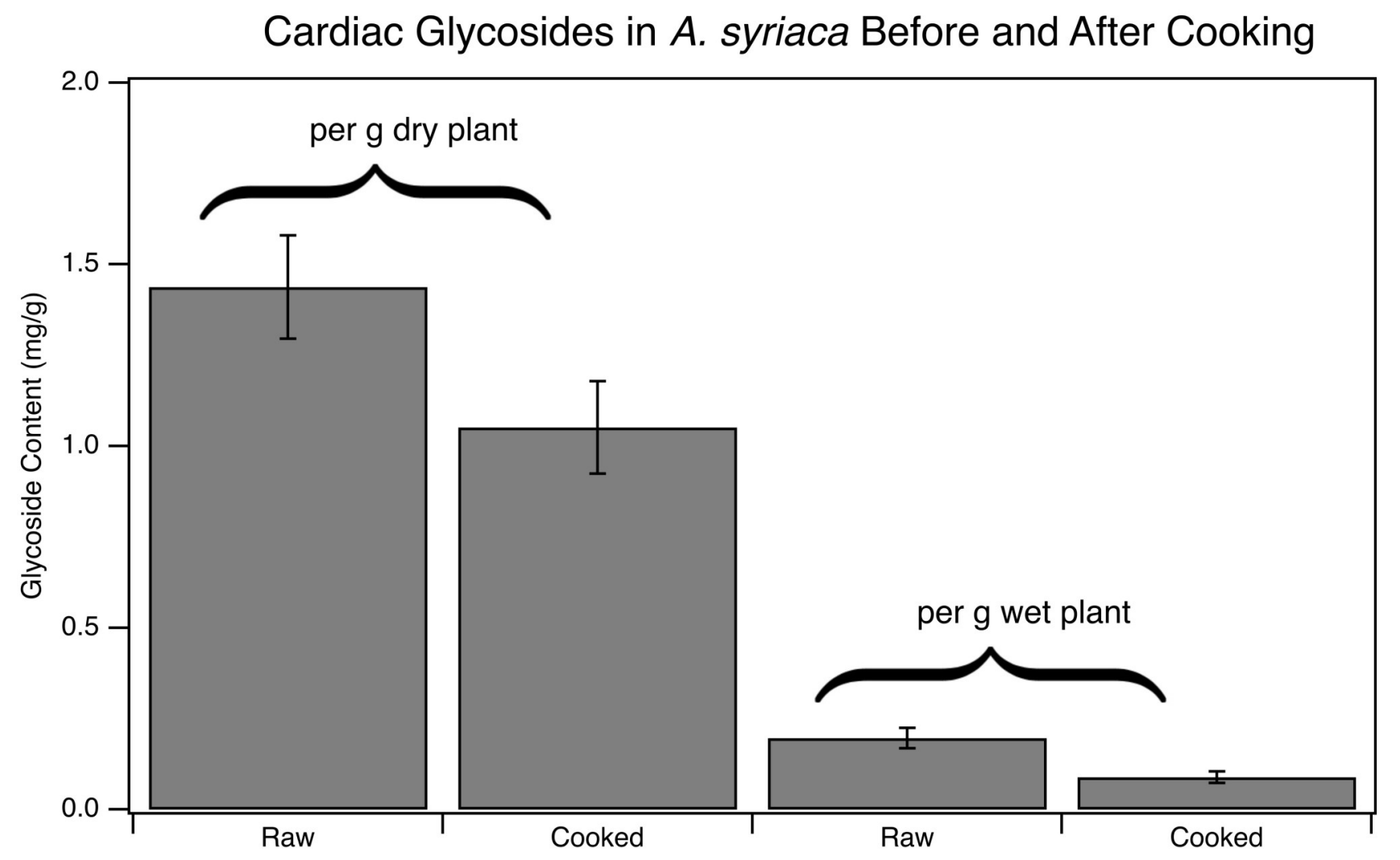

Figure 3 Amount of cardiac glycosides in A. syriaca before ("Raw") and after ("Cooked") cooking according to Myaamia tradition. Error bars are the $95 \%$ C.I.

on 7 raw plants and 34 measurements on 5 cooked plants. Three measurements were rejected because of visible detritus on or in the cuvettes during the spectrophotometric determination.

The cooked shoots are similar in appearance and texture to asparagus, for which a typical serving size is $90 \mathrm{~g}$. If one were to eat a similar size serving of $A$. syriaca cooked and harvested according to Myaamia tradition, one would be ingesting approximately $8 \mathrm{mg}$ of digitoxin-equivalent cardiac glycosides.

\section{Discussion}

We initially hypothesized that the Myaamia traditions pertaining to the timing of the harvest of $A$. syriaca were steering them toward plants that were in a stage of growth when they were less toxic. Specifically, because Myaamia only harvest relatively short, immature plants for food, similar to asparagus shoots, we expected to find lower amounts of glycoside in shorter plants compared to taller plants, and possibly that the stems contained less glycoside than the leaves. This initial expectation was only partly confirmed by the data. Although, as can been seen in Figure 1, the amount of glycoside does not correlate significantly with plant height for plants harvested when they are between 100 and $170 \mathrm{~mm}$ tall, and the small shoots were found to have significantly lower levels of cardiac glycosides than the stems and leaves of taller plants (Figure 2). If the Myaamia were to consume the stems of taller rather than the shorter, relatively immature shoots, they would be exposed to levels of glycoside levels significantly higher than what they get in their traditional practice.

Moreover, as shown in Figure 3, the abundance of glycosides in $A$. syriaca is further reduced by the traditional cooking procedure in which the plant material is repeatedly boiled in several portions of fresh water. The cooking procedure reduces the amount of cardiac glycoside in dry plant material to approximately two-thirds of the original value. Because cooked plant material also has a higher water content, the cooking procedure reduces the cardiac 
glycoside level in cooked plant material to about half of what it is in raw shoots.

However, the amount of cardiac glycosides in $A$. syriaca shoots that have been cooked according to Myaamia tradition still contains $0.1 \mathrm{mg}$ per gram undehydrated plant. Therefore, even a modest serving of $10 \mathrm{~g}$ of cooked plant material would contain $1 \mathrm{mg}$ of digitoxin-equivalent cardiac glycoside. This amount already exceeds the amount that might be prescribed by a physician for a heart condition, and is therefore physiologically active. According to the Hazardous Substances Data Bank, "healthy adults may develop symptoms after acute ingestions of more than 2 to 3 $\mathrm{mg}$, but rarely develop life-threatening toxicity with acute ingestions of less than $5 \mathrm{mg}$. Ingestions causing cardiac arrest in healthy adults are generally $10 \mathrm{mg}$ digoxin or more." Therefore, a serving of $100 \mathrm{~g}$ of cooked $A$. syriaca, despite having significantly lower levels of cardiac glycosides than the stems of plants, could potentially have sufficient glycoside to cause a serious medical issue.

Nonetheless, despite Gonella's (2007) ethnographic research, we are unaware of any reports of ill effects suffered by Myaamia who have consumed cooked A. syriaca. This discrepancy could be explained in several ways. First, through the use of the shoots for food over generations, they may, as a population, have become more resistant to the physiological effects than other populations. We doubt this to be the case because Myaamia have, on occasion, shared this food practice with non-Myamia individuals (including M. Gonella, one of the authors), and there are no reports of any ill effects on nonMyaamia individuals. Second, the toxicology data may be wrong. We doubt this to be the case because there are other documented reports of small amounts of cardiac-glycoside containing plants causing serious injury or death. Third, the negative effects of the toxins may be mitigated because the shoots are used as food for a very short period of the yearapproximately two weeks-so the total quantity ingested is limited temporally. Finally, our measured values may be upper-bounds on the amount of glycoside, rather than an exact determination, owing to limitations of the TNDP assay. There could be some poorly understood optical or chemical interference from the highly-colored background or latex precursors. For example, there could be a nonphysiologically active glycoside or other butenolide containing compound present in A. syriaca that is capable of reacting with TNDP and absorbing at our wavelength of interest. This final hypothesis could be confirmed or amended by repeating the analysis with a complementary analytical method, such as liquid chromatography (Ikeda et al. 1995), LC-MS (Grosa et al. 2005; Tor et al. 2005; Wang et al. 2000), or more sophisticated methods (Li et al. 2010).

\section{Conclusions}

There appear to be several aspects of Myaamia traditional use of $A$. syriaca that prevent eaters from receiving as much cardiac glycoside as is present in raw plants. The first is that they only harvest and use the shoots, which have roughly half of the glycoside content of taller-stemmed plants. Furthermore, the traditional cooking procedure removes approximately half of the cardiac glycosides that are present in uncooked shoots.

\section{Acknowledgments}

Several students made significant contributions to the early efforts of this project, including Claire Berchiolli, Chris Schene, Clay Garris, Erin LeVoir, Kalvyn Ngo, Sarah Clark Pierson, and Shane Rowan. This work has been done in cooperation with the Myaamia Center at Miami University. We are grateful for their support and encouragement to pursue this research.

\section{Declarations}

Permissions: None declared.

Sources of funding: MAE was supported by the Nishimura Faculty Research Fund and other Professional Development Grants at Westmont College. MPG was supported by the Miami Tribe of Oklahoma. HJB and JRW were supported by the Westmont College Department of Chemistry.

Conflicts of Interest: None declared.

\section{References Cited}

Araya, J. J., K. Kindscher, and B. N. Timmermann. 2012. Cytotoxic Cardiac Glycosides and Other Compounds from Asclepias syriaca. Journal of Natural Products. 75:400-407. DOI:10.1021/np2008076.

Brower, L. P., P. B. McEvoy, K. L. Williamson, and M. A. Flannery. 1972. Variation in Cardiac Glycoside Content of Monarch Butterflies from Natural Populations in Eastern North America. Science 177:426-429. DOI:10.1126/ science.177.4047.426. 
Cheatham, S., and M. C. Johnston. 1995. Useful Wild Plants of Texas, the Southeastern and Southwestern United States, the Southern Plains, and Northern Mexico. Useful Wild Plants, Austin, TX.

Densmore, F. 1974. How Indians Use Wild Plants for Food, Medicine and Crafts. Dover Publications, New York.

Gaertner, E. E. 1979. The History and Use of Milkweed (Asclepias syriaca L.). Economic Botany 33:119-123. DOI:10.1007/BF02858278.

Gilmore, M. R. 1911. Uses of Plants by the Indians of the Missouri River Region. Annual Report of the Bureau of American Ethnology 33:1911-12.

Gonella, M. P. 2007. Myaamia Ethnobotany. Unpublished Doctoral Dissertation, Department of Botany, Miami University, Oxford, $\mathrm{OH}$.

Grosa, G., G. Allegrone, and E. Del Grosso. 2005. LC-ESI-MS/MS Characterization of StrophanthinK. Journal of Pharmacentical and Biomedical Analysis 38:79-86. DOI:10.1016/j.jpba.2004.12.008.

Hamel, P. B., and M. U. Chilotsky. 1975. Cherokee Plants: Their Uses-A 400 Year History. Herald Publishing Co., Sylva, NC.

Ikeda, Y., Y. Fujii, I. Nakaya, and M. Yamazaki. 1995. Quantitative HPLC Analysis of Cardiac Glycosides in Digitalis purpurea Leaves. Journal of Natural Products 58:897-901. DOI:10.1021/np50120a012.

Li, Y., X. Wu, J. Li, Y. Wang, S. Yu, H. Lv, J. Qu, Z. Abliz, J. Liu, Y. Liu, and D. Du. 2010. Identification of Cardiac Glycosides in Fractions from Periploca forrestii by High-Performance Liquid Chromatography/Diode-Array Detection/Electrospray Ionization Multi-Stage Tandem Mass Spectrometry and Liquid Chromatography/Nuclear Magnetic Resonance. Journal of Chromatography B 878:381-390. DOI:10.1016/j.jchromb.2009.12.008.
Moerman, D. 1988. Native American Medicinal Plants: an Ethobotanical Dictionary. Timber Press Inc., Portland, OR.

Rasmann, S., A. A. Agrawal, S. C. Cook, and A. C. Erwin. 2009. Cardenolides, Induced Responses, and Interactions Between Above- and Belowground Herbivores of Milkweed (Asclepias spp.). Ecology 90:2393-2404. DOI:10.1890/08-1895.1.

Schlegel, V., R. Zbasnik, T. Gries, B. H. Lee, T. Carr, J.-Y. Lee, C. Weller, and S. Cuppett. 2011. Characterisation of Potential Health Promoting Lipids in the Co-Products of De-Flossed Milkweed. Food Chemistry 126:15-20. DOI:10.1016/ j.foodchem.2010.10.024.

Tor, E. R., M. S. Filigenzi, and B. Puschner. 2005. Determination of Oleandrin in Tissues and Biological Fluids by Liquid ChromatographyElectrospray Tandem Mass Spectrometry. Journal of Agricultural and Food Chemistry 53:4322-4325. DOI:10.1021/jf050201s.

Wang, X., J. B. Plomley, R. A., Newman, and A. Cisneros. 2000. LC/MS/MS Analyses of an Oleander Extract for Cancer Treatment. Analytical Chemistry 72:3547-3552. DOI:10.1021/ac991425a.

Whitford, A. C. 1941. Textile Fibers Used in Easterm Aboriginal North America. American Museum of Natural History, New York. 